\title{
Dipole-fiber systems: radiation field patterns, effective magnetic dipoles, and induced cavity modes
}

\author{
Shaghik Atakaramians ${ }^{\mathrm{a}}$, Andrey E. Miroshnichenko ${ }^{\mathrm{b}}$, Ilya V. Shadrivov ${ }^{\mathrm{b}}$, Tanya M. Monro ${ }^{\mathrm{c}, \mathrm{d}}$, \\ Yuri S. Kivshar ${ }^{\mathrm{b}}$, and Shahraam Afshar V., d \\ anstitute of Photonics and Optical Science, School of Physics, The University of Sydney, NSW \\ 2006, Australia \\ ${ }^{\mathrm{b} N o n l i n e a r}$ Physics Centre, Research School of Physics and Engineering, The Australian \\ National University, Canberra ACT 2601, Australia \\ ${ }^{\mathrm{c}}$ Laser Physics and Photonic Devices Laboratories, School of Engineering, University of South \\ Australia, Mawson Lake SA 5095, Australia \\ ${ }^{\mathrm{d} I n s t i t u t e}$ for Photonics and Advanced Sensing, The University of Adelaide, SA 5005, Australia
}

\begin{abstract}
We study the radiation patterns produced by a dipole placed at the surface of a nanofiber and oriented perpendicular to it, either along the radial ( $r$-oriented) or azimuthal ( $\phi$-oriented) directions. We find that the dipole induces an effective circular cavity-like leaky mode in the nanofiber. The first radiation peak of the $\phi$-oriented dipole contributes only to TE radiation modes, while the radiation of the $r$-oriented dipole is composed of both $\mathrm{TE}$ and TM radiation modes, with a relative contribution depending on the refractive index of the nanofiber. We reveal that the field pattern of the first resonance of a $\phi$-oriented dipole is associated with a magnetic dipole mode and strong magnetic response of an optical nanofiber.
\end{abstract}

Keywords: dipole-fiber system, whispering gallery modes, induced magnetic dipole

\section{INTRODUCTION}

The interaction between quantum emitters and electromagnetic waves in cavities, recognized as cavity quantum electrodynamics (CQED), ${ }^{1,2}$ has been explored for weak and strong coupling regimes for different cavities, ${ }^{3}$ such as microspheres, ${ }^{4-6}$ microtoroids ${ }^{7,8}$ and microdisks. ${ }^{9}$ The modification of different aspects of the emission of quantum emitters, such as radiation peaks, in the vicinity of dielectric waveguides have also been considered. ${ }^{10-18}$ Radiation peaks in the emission of quantum emitters in the vicinity of optical fibers have been observed experimentally ${ }^{10}$ and studied theoretically being associated with the whispering gallery modes (WGMs) of fibers. ${ }^{15,17,18}$ The coupling of the emission from quantum emitters into guided and radiation modes of optical fibers has been studied, ${ }^{10,13,15-17,19-24}$ and identified as a potential platform for developing devices for quantum information science. ${ }^{20,23,24}$

Here, we investigate the radiation modes and field patterns of a dipole-fiber system where the dipole is placed perpendicular to the fiber with two different orientations, either along the radial (the so-called $r$-oriented dipole) or azimuthal (the so-called $\phi$-oriented dipole) directions. We show that the field pattern clearly indicates the formation of induced radial cavity modes which overlap with the positions of TM-polarized whispering gallery modes. In addition, we find that the first radiation peak of the $\phi$-oriented dipole has contribution only from TE radiation mode, while the first peak of the $r$-oriented dipole-fiber system has strong contribution of both TE and TM radiation modes. The relative contribution of TE and TM radiation modes in the system depends on the refractive index of the fiber. We reveal that the field pattern of the first resonance of a $\phi$-oriented dipole is associated with a magnetic dipole mode and strong magnetic response of an optical nanofiber.

Further author information: (Send correspondence to S.A.)

S.A.: E-mail: shaghik.a@sydney.edu.au

SPIE Micro+Nano Materials, Devices, and Systems, edited by Benjamin J. Eggleton, Stefano Palomba Proc. of SPIE Vol. 9668, 96683J · @ 2015 SPIE · CCC code: 0277-786X/15/\$18 · doi: 10.1117/12.2204783 


\section{ANALYTICAL RESULTS}

We consider an optical system consisting of an electric dipole and air-clad fiber, as shown in Fig. 1. The electric dipole is located on the core-clad interface and the fiber is oriented along $z$-axis. We assume that the dipole emits at a single wavelength $(\lambda=700 \mathrm{~nm})$ and telluride $(n=2.02)$ is the hosting material of the fiber. It was shown that a z-oriented dipole-fiber system have strong resonance peaks in radiated power, which are associated with the TE whispering gallery modes (WGMs) formed in the cross section of the fiber. ${ }^{18}$

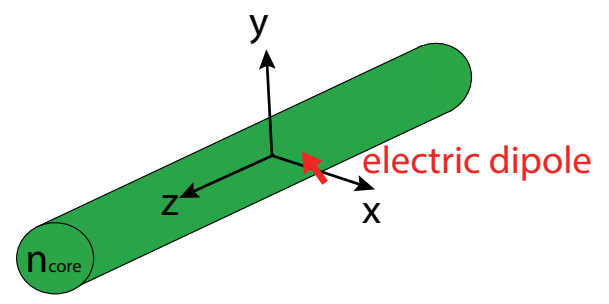

Figure 1. Schematic diagram of electric dipole and fiber system.

To find the total radiated power, one can start from the total electric field of the system. ${ }^{18}$ The total electric field of the dipole-waveguide system can be expanded using $z$-propagating plane waves as: ${ }^{18}$

$$
\mathbf{E}(x, y, z)=\sum_{j} a_{j} \mathbf{e}_{j}(x, y) e^{i \beta_{j} z}+\sum_{j} \int_{0}^{Q_{\max }} a_{j}(Q) \mathbf{e}_{j}(x, y, Q) e^{i \beta(Q) z} d Q+B K,
$$

in which $n_{c o}$ and $n_{c l}$ are the refractive indices of the core and cladding, respectively, $a_{j}, a_{j}(Q)$ and $\mathbf{e}_{j}(x, y)$, $\mathbf{e}_{j}(x, y, Q)$ are coupling coefficients and modal vector fields for forward guided and radiation modes, respectively, $\beta_{j}$ 's are the corresponding propagation constants, $Q=(D / 2)\left(k^{2} n_{c l}^{2}-\beta^{2}\right)^{1 / 2}, D$ is the core diameter, $k=2 \pi / \lambda$, and $B K$ represents the contribution of backward guided and radiation modes. ${ }^{23,25}$ In general, guided and radiation modes are orthogonal to each other in the sense that $\int_{A_{\infty}}\left(\mathbf{e}_{i} \times \mathbf{h}_{j}^{*}\right) \cdot \hat{z} d A=2 N_{i} \delta_{i j}$ and the discrete and continuous values of the propagation constants $\beta$ of these modes, respectively, are bounded by: ${ }^{25} k_{c l} \leq \beta_{j} \leq k n_{c o}$ for guided modes and $0 \leq \beta \leq k n_{c l}$ for radiation modes. The coupling coefficients $a_{j}$ are:

$$
\left|a_{j}\right|^{2}=\frac{\omega^{2}}{16 N_{j}^{2}}\left|\mathbf{e}_{j}^{*}\left(\mathbf{r}_{0}\right) \cdot \mathbf{p}_{0}\left(\mathbf{r}_{0}\right)\right|^{2},
$$

with a similar equation for $a_{j}(Q)$. Here $\omega$ is the transition frequency of the dipole, $\mathbf{p}_{0}$ is the dipole moment with the strength of $p_{0}$, and $N_{j}=(1 / 2) \int_{A_{\infty}}\left(\mathbf{e}_{j} \times \mathbf{h}_{j}^{*}\right) \cdot \hat{z} d A$ is a normalization term. The power captured in each mode is $P_{j}=\left|a_{j}\right|^{2} N_{j}$ and hence the total emitted power of the dipole-waveguide system is the sum of the power captured into all guided and radiation modes, i.e.

$$
P_{\text {total }}=\sum_{j} P_{j}+\sum_{\nu} \int_{0}^{Q_{\max }} P_{\nu}(Q) d Q
$$

The normalized radiated power for a $\phi$ - and $r$-oriented dipole excitations (cylindrical coordinates) are shown in Figs. 2 (a) and (b). The total radiated power is normalized to radiated dipole power in telluride glass, $P_{T e}=\mu_{0} p_{0}^{2} \omega^{4} n / 12 \pi c$. Due to the normalization to $P_{T e}$, the results shown in Fig. 2 are independent of the dipole strength $p_{0}$. Note that the ratio of the radiation power to $P_{T e}$ is equal to the Purcell factor. It can be seen that for an $r$-oriented dipole the enhancement is very strong (more than 2 for the first radiation peak).

We investigate the physics behind the radiation peaks by using an independent method that explains the formation of WGMs in a 2D circular disk, which is a representation of a thin cross section of the fiber. ${ }^{18}$ For such a disk, TE (with non-zero components; $\mathbf{E}_{z}, \mathbf{H}_{r}$, and $\mathbf{H}_{\theta}$ ) and TM (with non-zero components; $\mathbf{H}_{z}, \mathbf{E}_{r}$, and 

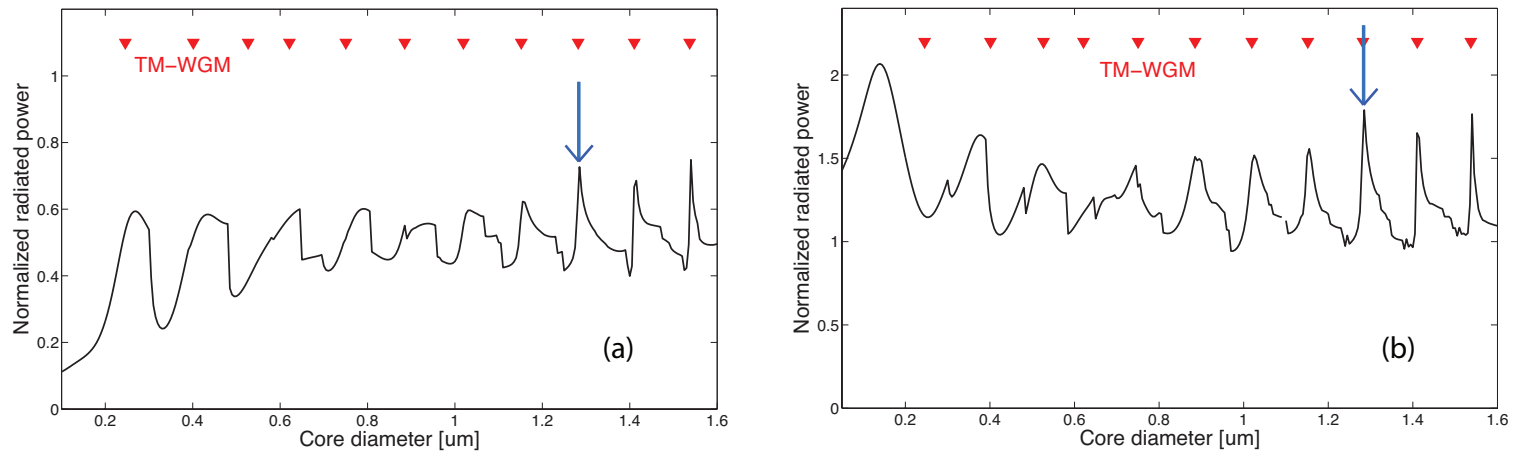

Figure 2. Normalized total radiated power of a dipole-fiber system with (a) $\phi$-, and (b) $r$-oriented excitation. All the radiated powers are normalized to the dipole radiation power in bulk tellurite glass. The radius of the $(m, 1)$ resonances of $2 \mathrm{D}$ TM-WGMs are shown with red triangles.

$\mathbf{E}_{\theta}$ ) solutions of Maxwell's equations, for electromagnetic fields propagating around the disk, have the dispersion relations;

$$
J_{m}^{\prime}\left(n_{c o} x\right) H_{m}\left(n_{c l} x\right)-q_{s} H_{m}^{\prime}\left(n_{c l} x\right) J_{m}\left(n_{c o} x\right)=0
$$

where $x=k D / 2, J_{m}$ and $H_{m}$ are the Bessel and Hankel functions, and $q_{s}=\left(n_{c l} / n_{c o}\right)$ for TE and $q_{s}=\left(n_{c o} / n_{c l}\right)$ for TM modes. Solutions of Eq.(4) determine the location of WGM resonances ( $k D / 2$ values) that can be labeled by $(m, p)$, where $m$ and $p$ are the azimuthal and radial mode numbers, respectively.

We observe that the fundamental $(m, 0)$ TM-WGM resonances at $\lambda=700 \mathrm{~nm}$ overlap nicely with the radiation peaks of the $\phi$-oriented dipole-fiber system, Fig.2 (a), which have been obtained independently through Eqs. (4). While, for an $r$-oriented dipole-fiber system it nicely overlaps for $m>4$ resonances. To investigate the reason for discrepancy of the location of the peaks and TM-WGM resonance, we looked into the contribution of TM and TE raidaited components. The radiation modes are divided into TE- and TM-like elementary modes, ${ }^{25}$ modes where respectively the longitudinal electric and magnetic fields are zero or negligible. The total radiation power is the summation of the TE and TM radiation mode powers. ${ }^{23,25}$

The normalized radiated power and the contribution of TE and TM components form a $\phi$ - and $r$-oriented dipole excitation are shown in Figs. 3 (a)-(c). When the electric dipole source is oriented along $\phi$ direction, the total radiation has a dominant TE component, which defines the position of the resonance peaks [see Fig. 3(a)]. A $\phi$-oriented dipole has a magnetic field oriented along $z$ direction in the $x y$-plane leading to excitation of the radiated modes with $z$-component of magnetic field, i.e. mainly the TE modes. When the electric dipole source is oriented along $r$ direction (Figs. 3 (b) and (c)), the four first radiation peaks have both TE and TM radiation contribution. An $r$-oriented dipole has a magnetic field oriented along $z$ direction in the $x y$-plane and an electric field along $z$ direction in the $x z$-plane leading to excitation of both TE and TM radiated modes respectively. As a result, the four first radiation peaks do not align with the position of the WGM resonances due to existence of both components. Comparison of Figs. 3 (b) and (c), where the refractive index of the fiber is different, shows that the contribution of TE and TM components depend on the refractive index of the fiber for the $r$-oriented dipole. This is due to higher concentration of electric field component in the higher dielectric medium.

\section{NUMERICAL MODELING}

In order to verify the formation of whispering gallery modes in the system, we modelled our structure using CST microwave studio. We use an electric dipole excitation as the source. We use open boundary conditions (waves passing through the boundary with minimum reflection, i.e. perfectly matched layer) in the $z$-direction and "open (add space)" boundary conditions (similar to open with an extra space) in $x$ - and $y$-directions, to represents infinitely long fiber along $z$-axis. We examine the dipole-fiber system at the position of the eight 

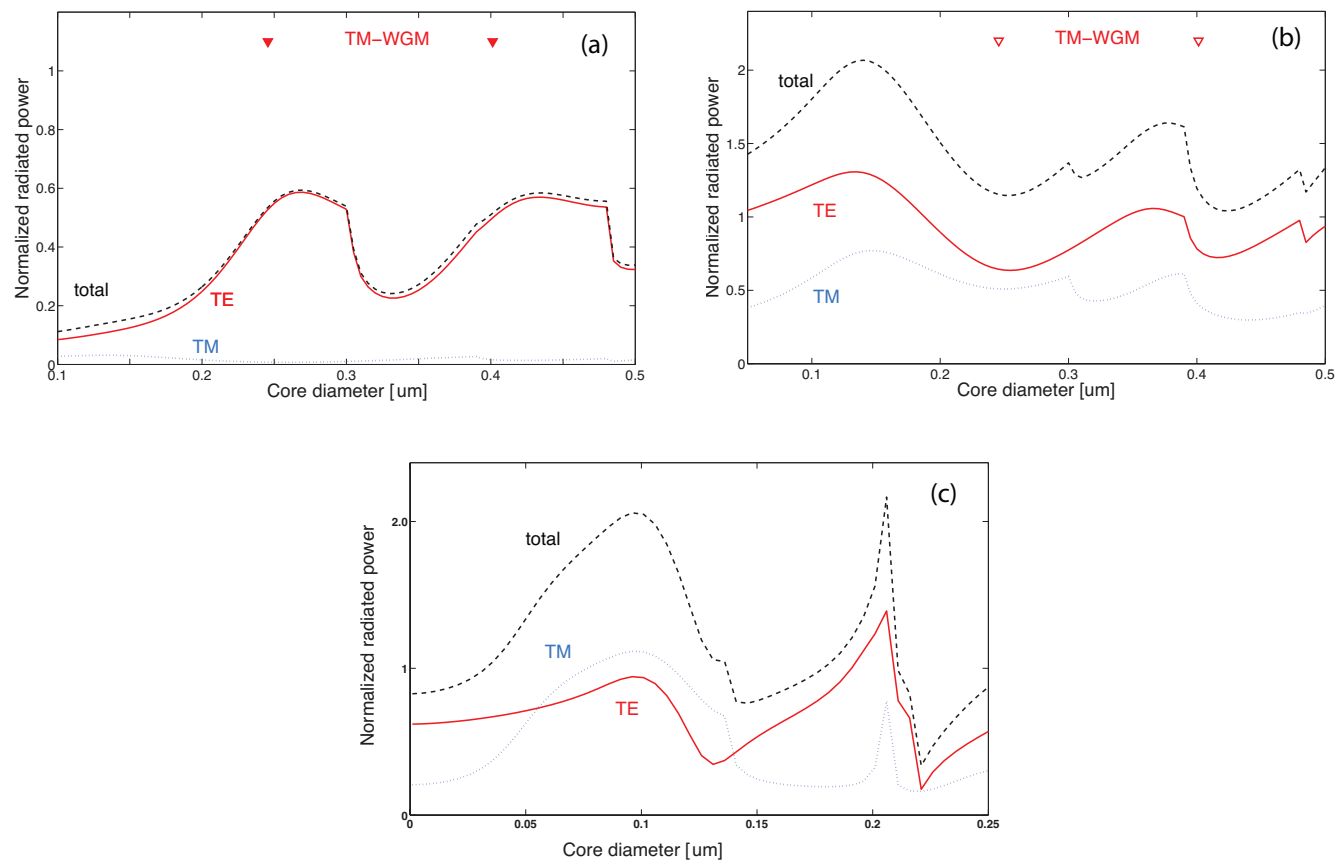

Figure 3. Normalized total radiated power (black dashed line) and the contribution of TM (blue dotted line) and TE (red solid line) radiated power of a dipole-fiber system with (a) $\phi_{-}$, (b) and (c) $r$-oriented excitation. The refractive index of the fiber is $n_{\text {core }}=2.05$ for parts (a) and (b), while it is $n_{\text {core }}=4$ for part (c). The radius of the $(m, 0)$ resonances of 2D TM-WGMs are shown with red triangles.

radiation peak, shown with blue arrows in Fig. 2. The position of these radiation peaks overlaps with TE WGM $(8,0)$ for $z$ orientation and TM WGM $(8,0)$ for $\phi$ and $r$ orientations. Figure 4 (a) shows the z-component of electric field in the cross-section of the fiber. It confirms the formation of $\mathrm{TE}(8,0)$ whispering gallery mode in the fiber cross-section. Figures 4 (b) and (c) shows the z-component of magnetic field in the cross-section of the fiber. It confirms the formation of $\operatorname{TM}(8,0)$ whispering gallery mode in the fiber cross-section when dipole is oriented along $\phi$ and $r$, respectively. Figures 4 (d) and (e) show the 3D electric field patterns for $z$-oriented dipole system respectively at the position of the second and eight radiation peak. These verifies that for higher order modes (Fig. 4 (e)) the radiation is localized within a transverse plane normal to the fiber axis and at the position of the dipole, i.e. the peak is mainly due to contributions from a very small range of non-transverse propagating modes (skew modes) compared to that of low order resonances (Fig. 4 (d)). Note that the dipole is located on the core-clad interface on the right hand side of the fiber, shown with black circle in Figs. 4 (a)-(c).

We also investigate the magnetic and electric fields at the position of the first peak for each orientation of dipole. We noticed that when electric dipole is along $\phi$ direction the magnetic field inside the fiber makes a loop as shown in Fig. 5. This indicates formation of a magnetic dipole along the fiber, which is induced due to $z$-component of the magnetic field of the excitation.

\section{CONCLUSIONS}

We have studied the radiation patterns of an $r$ - and $\phi$-oriented dipole-fiber system and observed that the dipole induces radial cavity modes which overlaps with position of whispering gallary modes. Moreover, we have found that the first radiation peak of the $\phi$-oriented dipole has contribution only from TE radiation modes, while that of the $r$-oriented dipole-fiber system has strong contribution from both TE and TM radiation modes. Furthermore, we have demonstrated the formation of WGM using numerical modelling and showed that the field pattern of the first resonance of a $\phi$-oriented dipole-fiber system corresponds to an effective magnetic response. 


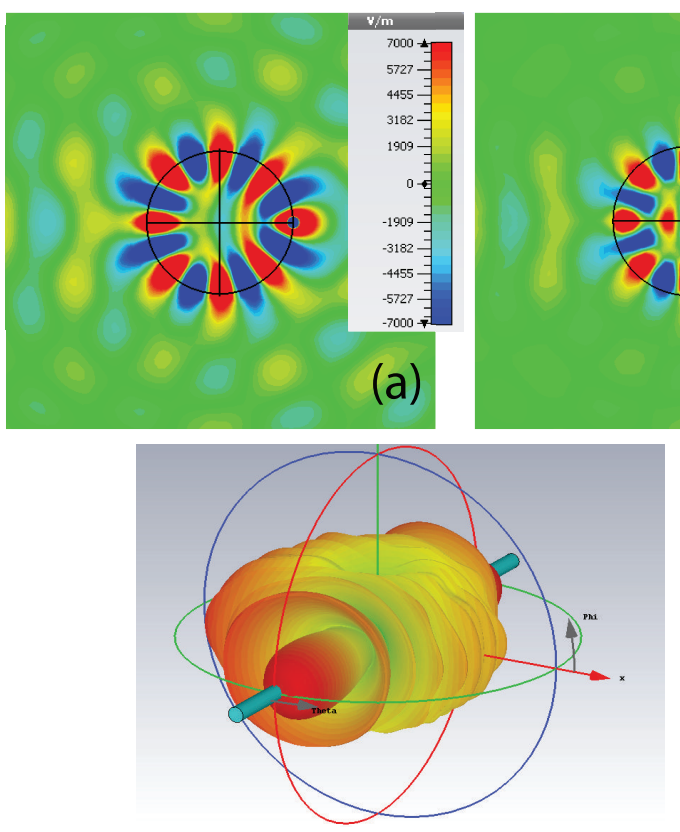

(d)

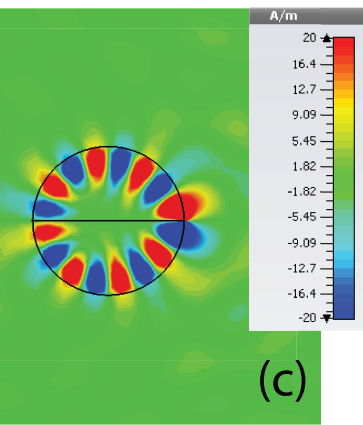

(b)

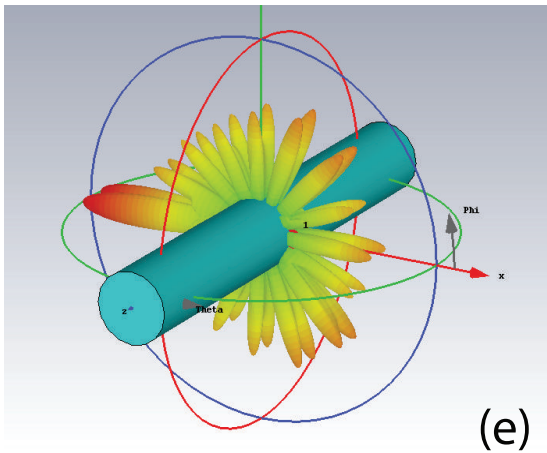

Figure 4. (a) $E_{\mathrm{z}}$ component of the electric field in $z=0$ plane, when dipoe is orieneted along the $z$ diretion. (b) and (c) $H_{\mathrm{z}}$ component of the magnetic field in $z=0$ plane, when dipole is orieneted along the $\phi$ and $r$ diretions, respectively. The black circle represents the core-clad interface. The diameter of the fiber is $1.1 \mu \mathrm{m}, 1.285 \mu \mathrm{m}$ and $1.285 \mu \mathrm{m}$ respectively for parts (a), (b) and (c), which overlaps with $(8,0)$ resonances of 2D TE-WGM for part (a) and TM-WGM for parts (b) and (c). The dipole is located on the core-clad interface on the right hand side of the fiber, shown with black circle. The $3 \mathrm{D}$ electric field patterns for $z$-oriented dipole system at the position of the (d) second and (e) eight radiation peaks.

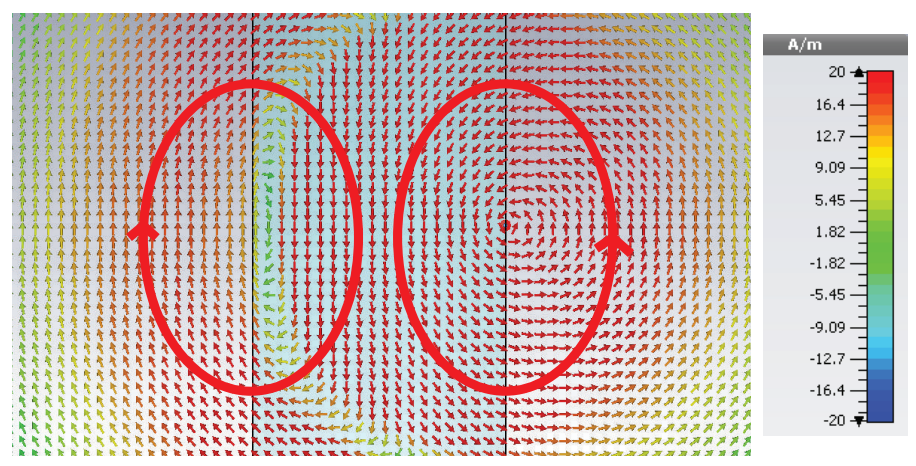

Figure 5. $\quad H_{\mathrm{z}}$ component of the magnetic field in $y=0$ plane, when dipole is orieneted along the $\phi$ diretions. The induced magnetic dipole is highlighted with red elliptical shapes.

\section{ACKNOWLEDGMENTS}

S. Atakaramians acknowledges a support of the Australian Research Council (ARC) through the project DE140100614. T. M. Monro acknowledges the support of an ARC Georgina Sweet Laureate Fellowship.

\section{REFERENCES}

[1] Purcell, E. M., "Spontaneous emission probabilities at radio frequencies," Phys. Rev. 69, 681 (1946).

[2] Berman, P., [Cavity Quantum Electrodynamics], Academic Press, 1st edition ed. (Dec. 1993).

[3] Vahala, K. J., "Optical microcavities," Nature 424, 839-846 (Aug. 2003). 
[4] Vernooy, D., Furusawa, A., Georgiades, N., Ilchenko, V., and Kimble, H., "Cavity QED with high- $\$ Q \$$ whispering gallery modes," Physical Review A 57, 2293-2296 (1998).

[5] Schniepp, H. and Sandoghdar, V., "Spontaneous Emission of Europium Ions Embedded in Dielectric Nanospheres," Physical Review Letters 89, 257403 (2002).

[6] Spillane, S., Kippenberg, T., Painter, O., and Vahala, K., "Ideality in a Fiber-Taper-Coupled Microresonator System for Application to Cavity Quantum Electrodynamics," Physical Review Letters 91, 2-5 (2003).

[7] Armani, D. K., Kippenberg, T. J., Spillane, S. M., and Vahala, K. J., "Ultra-high-q toroid micro cavity on an chip," Nature 421(6926), 925-928 (2003).

[8] Aoki, T., Dayan, B., Wilcut, E., Bowen, W. P., Parkins, A. S., Kippenberg, T. J., Vahala, K. J., and Kimble, H. J., "Observation of strong coupling between one atom and a monolithic microresonator," Nature 443, 671-674 (Oct. 2006).

[9] Kiraz, A., Michler, P., Becher, C., Gayral, B., Imamolu, A., Zhang, L., Hu, E., Schoenfeld, W. V., and Petroff, P. M., "Cavity-quantum electrodynamics using a single InAs quantum dot in a microdisk structure," Applied Physics Letters 78, 3932-3934 (June 2001).

[10] Owen, J. F., Barber, P. W., Dorain, P. B., and Chang, R. K., "Enhancement of Fluorescence Induced by Microstructure Resonances of a Dielectric Fiber," Physical Review Letters , 1075-1078 (1981).

[11] Heinzen, D. L. and Feld, M. S., "Vacuum Radiative Level Shift and Spontaneous-Emission Linewidth of an Atom in an Optical Resonator," Physical Review Letters 59, 2623-2626 (1987).

[12] Björk, G., Machida, S., Yamamoto, Y., and Igeta, K., "Modification of spontaneous emission rate in planar dielectric microcavity structures," Physical Review A 44, 669-681 (1991).

[13] Chu, D. Y. and Ho, S.-T., "Spontaneous emission from excitons in cylindrical dielectric waveguides and the spontaneous-emission factor of microcavity ring lasers," J. Opt. Soc. Am. B, JOSAB 10, 381-390 (Feb. 1993).

[14] Janowicz, M. and Zakowicz, W., "Quantum radiation of a harmonic oscillator near the planar dielectricvacuum interface," Physical Review A 50, 4350-4364 (1994).

[15] Zakowicz, W. and Janowicz, M., "Spontaneous emission in the presence of a dielectric cylinder," Physical Review A 62, 013820 (2000).

[16] Klimov, V. V. and Ducloy, M., "Spontaneous emission rate of an excited atom placed near a nanofiber," Physical Review A 69, 013812 (2004).

[17] Fussell, D. P., McPhedran, R., and de Sterke, C. M., "Decay rate and level shift in a circular dielectric waveguide," Physical Review A 71, 013815 (2005).

[18] Afshar V, S., Henderson, M. R., Greentree, A. D., Gibson, B. C., and Monro, T. M., "Self-formed cavity quantum electrodynamics in coupled dipole cylindrical-waveguide systems," Optics Express 22, 11301 (May 2014).

[19] Le Kien, F., Dutta Gupta, S., Balykin, V. I., and Hakuta, K., "Spontaneous emission of a cesium atom near a nanofiber: Efficient coupling of light to guided modes," Physical Review A 72, 032509 (Sept. 2005).

[20] Le Kien, F. and Hakuta, K., "Cavity-enhanced channeling of emission from an atom into a nanofiber," Physical Review A 80, 053826 (Nov. 2009).

[21] Nayak, K. P., Kien, F. L., Kawai, Y., Hakuta, K., Nakajima, K., Miyazaki, H. T., and Sugimoto, Y., "Cavity formation on an optical nanofiber using focused ion beam milling technique," Optics Express 19, 14040-14050 (July 2011).

[22] Henderson, M. R., Gibson, B. C., Ebendorff-Heidepriem, H., Kuan, K., Afshar V., S., Orwa, J. O., Aharonovich, I., Tomljenovic-Hanic, S., Greentree, A. D., Prawer, S., and Monro, T. M., "Diamond in Tellurite Glass: a New Medium for Quantum Information," Advanced Materials 23, 2806-2810 (July 2011).

[23] Henderson, M. R., Afshar V, S., Greentree, A. D., and Monro, T. M., "Dipole emitters in fiber: interface effects, collection efficiency and optimization," Optics Express 19(17), 16182-16194 (2011).

[24] Yalla, R., Le Kien, F., Morinaga, M., and Hakuta, K., "Efficient Channeling of Fluorescence Photons from Single Quantum Dots into Guided Modes of Optical Nanofiber," Physical Review Letters 109, 063602 (Aug. 2012).

[25] Snyder, A. W. and Love, J., [Optical Waveguide Theory], Springer, 1st edition ed. (1983). 